\title{
Characteristics of unconventional Rb magneto-optical traps
}

\author{
K. N. Jarvis, ${ }^{*}$ B. E. Sauer, and M. R. Tarbutt \\ Centre for Cold Matter, Blackett Laboratory, Imperial College London, Prince Consort Road, London SW7 2AZ, United Kingdom
}

(Received 19 July 2018; published 25 October 2018)

\begin{abstract}
We study several magneto-optical trapping configurations in ${ }^{87} \mathrm{Rb}$. These unconventional MOTs all use type-II transitions, where the angular momentum of the ground state is greater than or equal to that of the excited state. Some use red-detuned light, and others blue-detuned light. The properties of these MOTs are strongly influenced by the balance between opposing Doppler and Sisyphus forces, and vary widely from one configuration to another. In the blue-detuned MOT, Sisyphus cooling dominates over Doppler heating for all relevant speeds and magnetic fields. We measure the capture velocity of this MOT as a function of intensity and detuning, finding a maximum of $3.8 \pm 0.1 \mathrm{~m} / \mathrm{s}$. Atomic densities are particularly high in the blue-detuned MOT, and its lifetime is limited by collisions between the trapped atoms. We present measurements of the loss rate due to these ultracold collisions as a function of laser intensity and detuning. In the red-detuned MOTs, Sisyphus heating dominates at low speeds and Doppler cooling at higher speeds. Consequently, temperatures in the red-detuned MOTs are up to a thousand times higher than in the blue-detuned MOTs. One MOT forms large ring structures, with no density at the center, showing how atoms driven towards a non-zero equilibrium speed remain trapped by orbiting around the center. Another MOT demonstrates that magnetic mixing of the excited-state hyperfine levels can be an important mechanism in type-II MOTs.
\end{abstract}

DOI: 10.1103/PhysRevA.98.043432

\section{INTRODUCTION}

It has been over 30 years since the first demonstration of a magneto-optical trap (MOT) [1]. Since then, the MOT has led to a diverse range of applications and facilitated the emergence of broad fields of productive research. Normally, atomic MOTs operate on type-I transitions, where the hyperfine angular momentum of the excited state $\left(F^{\prime}\right)$ exceeds that of the ground state $(F), F^{\prime}=F+1$. Examples also exist of type-II atomic MOTs [2-5], which have $F^{\prime} \leqslant F$, but these have the unfavorable properties of high temperature and low density, and so have not received much attention. Recently, a number of groups have demonstrated magneto-optical trapping of diatomic molecules [6-8], which are cooled using type-II transitions [9], and this has generated a renewed interest in understanding the properties of type-II MOTs [10,11]. Those properties are strongly influenced by the balance between Doppler and Sisyphus forces, which always have opposite signs in type-II MOTs.

We recently proposed and demonstrated a MOT using blue-detuned light which relies exclusively on Sisyphus cooling [12]. This MOT performed exceptionally well, providing a phase-space density a million times higher than reported in any other type-II MOT, and even exceeding the phasespace density of most conventional type-I MOTs. This makes

\footnotetext{
*k.jarvis14@imperial.ac.uk

Published by the American Physical Society under the terms of the Creative Commons Attribution 4.0 International license. Further distribution of this work must maintain attribution to the author(s) and the published article's title, journal citation, and DOI.
}

the blue-detuned MOT particularly interesting for molecular MOTs, useful for loading atoms and molecules into conservative traps, and a good starting point for evaporation to quantum degeneracy. Here, we extend our study of the bluedetuned MOT. We measure its capture velocity as a function of laser intensity and detuning, and use these results to explain its stability. We also measure the loss rate coefficient due to ultracold collisions in this MOT for various intensities and detunings. Taken together, these measurements of capture velocity and loss rate provide useful information about the collisional processes in the MOT.

To extend further our study of type-II MOTs, we explore several additional magneto-optical trapping configurations using the $D_{2}$ line in ${ }^{87} \mathrm{Rb}$. We find eight new combinations of transitions that produce a stable MOT. We illustrate how significantly the properties of these traps can differ, both from one another and from the widely studied type-I MOT. The temperature of the atoms in various MOTs can differ by a factor of 1000 , and their lifetimes by a factor of 100 . In some cases, atoms form striking ringlike structures, orbiting around the center of the MOT because the balance between Doppler and Sisyphus forces drives them towards a non-zero equilibrium speed. We also find a MOT that appears to rely on mixing of the excited-state hyperfine levels by the magnetic field, as found previously in $\mathrm{Na}$ [4].

Our study helps improve the understanding of a ubiquitous and important technique used throughout the atomic physics community, and one that is emerging as a key tool for cooling and trapping molecules. These MOT configurations may also be valuable for studying ultracold collisions, especially the influence of hyperfine state and laser detuning. For example, we demonstrate configurations where most of the population resides in the lower hyperfine ground state, in contrast to 
the usual MOT, and configurations where the effects of nearresonant blue-detuned light on ultracold collisions can be studied.

\section{EXPERIMENT}

Our experimental setup consists of a simple vapor-cell magneto-optical trap. Two separate lasers provide the cooling and trapping light needed. One drives transitions from the lower hyperfine ground state, and is called $\mathcal{L}_{1}$, while the other drives transitions from the upper hyperfine ground state, and is called $\mathcal{L}_{2}$. Their detunings from the excited state $F^{\prime}$ are written $\Delta_{F F^{\prime}}$. The intensity at the center of the MOT is $I_{t}$ and is divided approximately equally between the six MOT beams and again between $\mathcal{L}_{1}$ and $\mathcal{L}_{2}$. A third laser, $\mathcal{L}_{\text {ref }}$, is locked to the $F=2 \rightarrow F^{\prime}=1,3$ saturated absorption crossover resonance of ${ }^{87} \mathrm{Rb}$. It is used as a frequency reference and for absorption imaging of the atoms. The frequencies of $\mathcal{L}_{1,2}$ are stabilized relative to $\mathcal{L}_{\text {ref }}$ using frequency-offset locks. The light from $\mathcal{L}_{1}$ and $\mathcal{L}_{2}$ is overlapped on a 50:50 beam-splitter cube, whose outputs are used to form two independent sets of MOT beams of opposite handedness. A pair of acousto-optical modulators (AOMs) is used to control whether each of the sets of beams is on or off. At the start of an experiment $\mathcal{L}_{2}$ is red-detuned from the transition to $F^{\prime}=3$, while $\mathcal{L}_{1}$ is tuned to either $F^{\prime}=1$ or $F^{\prime}=2$, both of which can function as repumping transitions. A type-I MOT then loads from the atomic vapor produced by a dispenser. Once this MOT is loaded, the intensities of both $\mathcal{L}_{1}$ and $\mathcal{L}_{2}$ are reduced and the detuning of $\mathcal{L}_{2}$ increased, which further cools the trapped atoms. Then, the light is quickly switched off using the AOM, the lasers are relocked to new frequencies, and light with the desired handedness is switched back on. Many of the MOTs described here require this precooling stage because the Doppler-cooling forces are too weak to capture atoms directly from the room-temperature vapor.

Figure 1 shows the various configurations for which we have observed a stable MOT. The first trapping scheme illustrated is the type-I MOT. The other configurations are grouped into pairs according to the transition driven by $\mathcal{L}_{1}$. Each pair has one configuration with $F=2 \rightarrow F^{\prime}=2$ and one with $F=2 \rightarrow F^{\prime}=1$. According to the rules for the choice of polarization given in [10], the position-dependent force for atoms in $F=2$ should change sign between these two configurations. We observe a stable MOT for all the configurations shown, implying that the majority of the confining force is due to transitions from the lower hyperfine ground state. This observation motivates our categorization of the MOTs. Table I summarizes some of the properties of the MOTs, which are described in more detail throughout the paper.

\section{BLUE-DETUNED MOTS}

We begin by discussing the trapping schemes that use blue-detuned light. The principle of a blue-detuned MOT is explained in detail in [12]. Briefly, when considering a twolevel atom or molecule there are two possible combinations of laser detuning and polarization which produce the same position-dependent force. When the upper-state $g$ factor is positive, and $F^{\prime}=F$ or $F^{\prime}=F+1$, the beams that push a
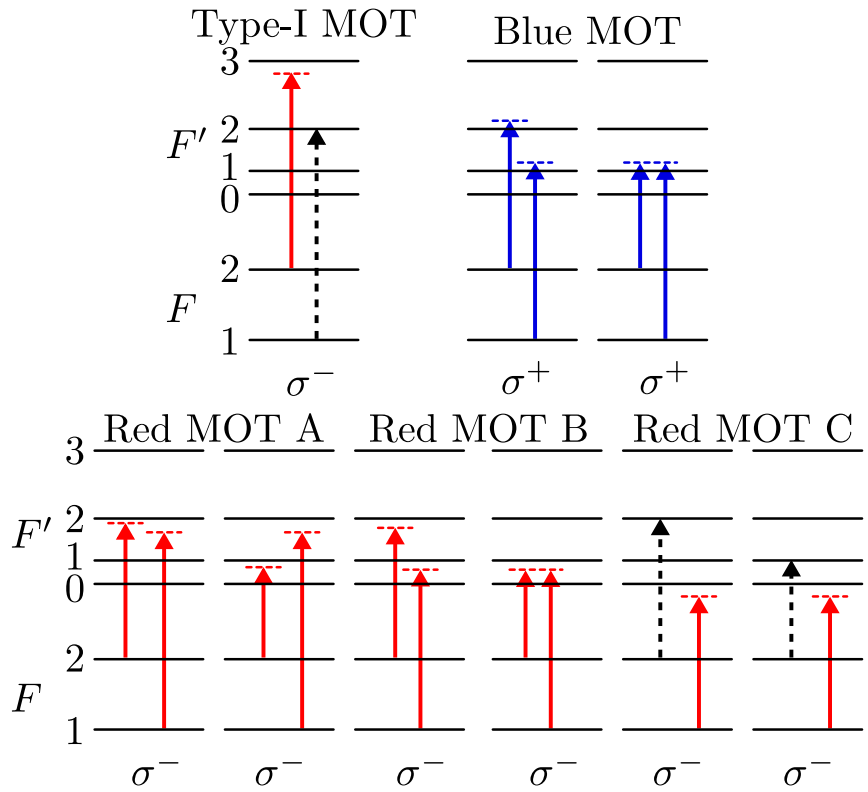

FIG. 1. Stable trapping configurations for cold atoms observed in this experiment. We choose the $z$ axis to be along the magnetic field direction. In each case the two lasers have the same polarization. In the type-I MOT when $g_{F^{\prime}}>0$, as in ${ }^{87} \mathrm{Rb}$, the laser beams that push displaced atoms back towards the center should be polarized to drive $\sigma^{-}$transitions. This is also true for red MOTs A, B and C. For the blue MOT, the polarizations are reversed. When a laser can be tuned to resonance with a transition without adversely affecting the MOT, the arrow representing the laser is colored black and shown dashed.

displaced atom back towards the center (which we call the restoring beams) can either be red-detuned and polarized to drive $\Delta m_{F}=-1$ transitions or it can be blue-detuned and polarized to drive $\Delta m_{F}=+1$. In type-II systems the Doppler and sub-Doppler velocity-dependent forces have the opposite sign for a given detuning. When the light is red-detuned, there is efficient Doppler cooling, so the capture velocity of the MOT is larger, but polarization-gradient forces heat the atoms to nonzero equilibrium velocities, so the temperature of atoms in a red-detuned MOT is high. Conversely, with blue-detuning there is efficient sub-Doppler cooling to low temperatures, but atoms moving faster than some critical velocity are heated by

TABLE I. Some properties of the various MOTs. Columns give the appearance, the temperature, the maximum lifetime, the approximate size, and whether or not the MOT can be loaded from the background (BG) vapor. Where the density distribution is Gaussian the quoted size is a typical value for the $1 / e^{2}$ radius. Otherwise the quoted size is indicative of the overall extent of the atom cloud. For MOTs B and C, the temperature was too high to measure using the usual ballistic expansion method, so we give only a lower bound.

\begin{tabular}{llrccc}
\hline \hline MOT & Appearance & $T(\mathrm{mK})$ & $\tau_{\max }(\mathrm{s})$ & Size $(\mathrm{mm})$ & BG \\
\hline Blue MOT $^{\mathrm{a}}$ & Gaussian & $0.02-0.2$ & 13 & 0.5 & No \\
Red MOT A & Gaussian & $10-25$ & 14 & 1 & Yes \\
Red MOT B & Ring-like & $>25$ & 9 & 5 & No \\
Red MOT C & Diffuse & $>25$ & 4 & 5 & No \\
\hline \hline
\end{tabular}

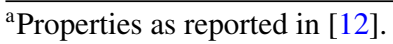


the Doppler forces and will evaporate from the MOT, resulting in a low capture velocity. In type-I systems, both Doppler and sub-Doppler cooling require red-detuned light, so no stable MOT exists when the light is blue-detuned.

We have observed two configurations of blue-detuned MOT. In the first configuration, presented in [12], $\mathcal{L}_{1}$ is detuned from $F^{\prime}=1$, and $\mathcal{L}_{2}$ is detuned from $F^{\prime}=2$. In the second combination, both lasers are detuned from $F^{\prime}=1$. In both configurations the two lasers are polarized so that the restoring beams drive $\Delta m_{F}=+1$ transitions. This is the opposite polarization to that used in the type-I MOT. For the MOT to work, $\mathcal{L}_{1}$ requires this polarization according to both the rate-equation model presented in [10] and the density-matrix model presented in [11]. When $\mathcal{L}_{2}$ is bluedetuned from $F^{\prime}=2$, the two approaches again agree that the restoring beam needs to drive $\Delta m_{F}=+1$ transitions. However, when blue-detuned from $F^{\prime}=1$, the rate-equation model predicts an anticonfining force for this polarization, whereas the density-matrix model predicts confinement for the range of magnetic fields explored by an atom close to the trap center. This difference in the nature of the confining force near the trap center was highlighted previously [11].

The properties of the two blue-detuned MOTs are similar to one another. Both produce clouds of a similar size. With $I_{t}=$ $400 \mathrm{~mW} / \mathrm{cm}^{2}, \Delta_{11} /(2 \pi)=+35 \mathrm{MHz}$, and $B^{\prime}=48 \mathrm{G} / \mathrm{cm}$ the temperature varies between 180 and $215 \mu \mathrm{K}$ as $\Delta_{21} /(2 \pi)$ is varied between 27 and $37 \mathrm{MHz}$. At a lower intensity, $I_{t}=$ $30 \mathrm{~mW} / \mathrm{cm}^{2}$, the temperature varies between 32 and $38 \mu \mathrm{K}$ as $\Delta_{21} /(2 \pi)$ is varied between 35 and $47 \mathrm{MHz}$. A blue-detuned MOT configuration was characterized in some detail in [12]. Here, we extend that study by measuring the capture velocity and the rate coefficient for trap loss due to ultracold collisions in the MOT.

\section{A. Capture velocity of the blue-detuned MOT}

The capture velocity, $v_{c}$, is an important parameter of a MOT that governs the steady-state trapped atom number and atom density through the loading rate of the trap $[13,14]$. Measurements of the capture velocity can also help us to understand which inelastic processes can lead to trap loss [15]. We measure the capture velocity using the following procedure. First, atoms are cooled and captured in the reddetuned type-I MOT and loaded into the blue-detuned MOT. Then, the MOT light is switched off using an AOM. Next, atoms are optically pumped into $F=2$ using a pulse of light resonant with the $F=1 \rightarrow F^{\prime}=2$ transition. Atoms are then launched horizontally through the center of the MOT by a push beam resonant with the $F=2 \rightarrow F^{\prime}=3$ cycling transition. The velocity of the atoms can be tuned by varying the push beam duration, and is calibrated by recording a series of absorption images of the moving atom cloud after the push is complete. From these images the center of mass velocity of the cloud is extracted. Once the atoms have been launched, the MOT is quickly switched back on and the atoms with $v<v_{c}$ are recaptured. After a short hold time the number of atoms in the MOT is recorded. This is repeated as the center-of-mass velocity of the cloud of atoms launched into the MOT is increased, from which the capture velocity of the trap can be inferred. Some data illustrating this are shown in
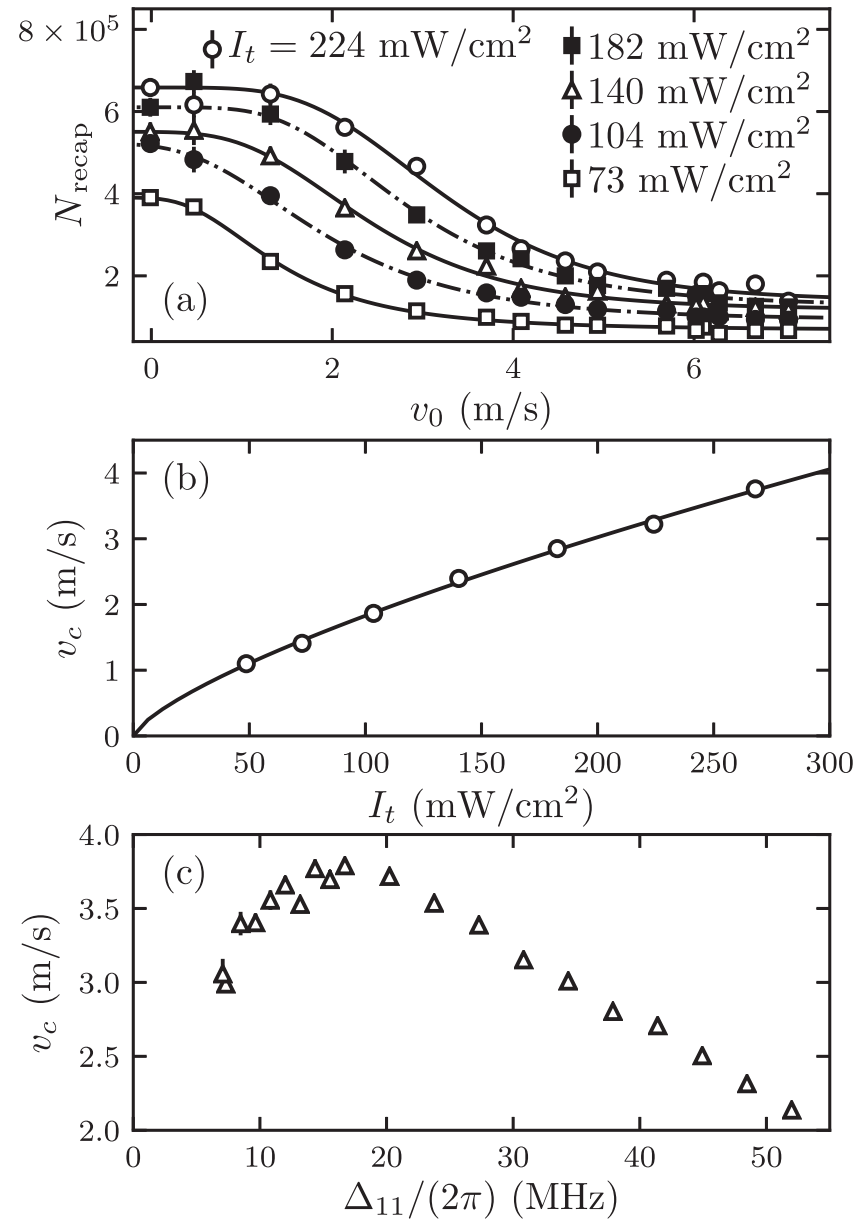

FIG. 2. Capture velocity, $v_{c}$, of the blue-detuned MOT. (a) Number of atoms recaptured by the MOT, $N_{\text {recap }}$, as a function of the center of mass velocity of the cloud, $v_{0}$. Data are shown for various trap intensities and each dataset is fit to extract the capture velocity at that intensity. (b) Capture velocity as a function of total trap intensity $I_{t}$. The data are fit to a polynomial $v_{c}=a I_{t}^{b}$, giving a best-fit exponent of $b=0.73(2)$. The other trap parameters are $\Delta_{11} /(2 \pi)=$ $+26 \mathrm{MHz}, \Delta_{22} /(2 \pi)=+11.5 \mathrm{MHz}$, and $B^{\prime}=39 \mathrm{G} / \mathrm{cm}$. (c) Capture velocity as a function of $\Delta_{11}$. The other parameters are $I_{t}=$ $260 \mathrm{~mW} / \mathrm{cm}^{2}$ and $B^{\prime}=39 \mathrm{G} / \mathrm{cm}$.

Fig. 2(a). For each of the sets of data the number of atoms recaptured, $N_{\text {recap }}$, is fit to a function

$$
\begin{aligned}
N_{\text {recap }}\left(v_{0}\right)= & N_{0}(F=1) \\
& +\frac{N_{0}(F=2)}{\sqrt{\pi} \sigma_{v_{0}}} \int_{-v_{c}}^{v_{c}} \exp \left[-\frac{\left(v-v_{0}\right)^{2}}{2 \sigma\left(v_{0}\right)^{2}}\right] d v,
\end{aligned}
$$

where $N_{0}(F)$ is the number of atoms in the ground-state hyperfine level labeled by $F$ immediately before the push, $v_{0}$ is the center of mass velocity of the atoms in $F=2$ after the push, $\sigma\left(v_{0}\right)$ characterizes the width of the velocity distribution after the atoms are launched, and $v_{c}$ is the capture velocity of the MOT along the trajectory of the launched atoms and is a free parameter in the fit. The velocity distribution is broadened by the gradient in the intensity of the push beam across the atom cloud, so its width $\sigma$ depends on $v_{0}$ through the push beam duration. This effect visibly skews the roll-off 
in the recaptured atom number, leading to the high-velocity tails in Fig. 2(a). To account for this, we find it sufficient to let the width $\sigma$ have a simple linear dependence on $v_{0}$, $\sigma\left(v_{0}\right)=\sigma_{0}+a v_{0}$, where $\sigma_{0}$ and $a$ are free parameters in the fit. In the measurements presented here atoms are held in the blue-detuned MOT for a total period of $200 \mathrm{~ms}$. Some atoms are lost during this period, and the fraction lost depends on the intensity. This is why $N_{\text {recap }}(0)$ depends on intensity. This has no effect on the measurement of $v_{c}$.

Figure 2(b) shows the capture velocity of the blue-detuned MOT as a function of $I_{t}$. The points are extracted from the best-fit parameters obtained from the curves in part (a). As expected, the capture velocity increases with increasing intensity. Fitting these data to the model $v_{c}=a I_{t}^{b}$ yields $b=0.73(2)$. Figure 2(c) shows how $v_{c}$ depends on the detuning, $\Delta_{11}$, when $I_{t}=260 \mathrm{~mW} / \mathrm{cm}^{2}$ and $B^{\prime}=39 \mathrm{G} / \mathrm{cm}$. The capture velocity has a maximum when $\Delta_{11} /(2 \pi) \approx 15 \mathrm{MHz}$. Across all parameters, the highest capture velocity measured is $v_{c}=3.8(1) \mathrm{m} / \mathrm{s}$. This is an order of magnitude smaller than is typical for a type-I MOT, reflecting the blue-detuned MOT's exclusive reliance on polarization-gradient forces. Nevertheless, over the whole range of the parameters explored, the capture velocity is at least 10 times higher than the mean velocity in the blue-detuned MOT [12], so the MOT is stable against the evaporative loss of atoms.

\section{B. Cold collisions in the blue-detuned MOT}

After loading the blue-detuned MOT, the number of trapped atoms, $N$, decays. We model this using the rate equation,

$$
\frac{d N}{d t}=-\gamma N-\beta \int n(\vec{r})^{2} d^{3} r
$$

where $\gamma$ is the one-body loss rate due to collisions with untrapped atoms, and $n(\vec{r})$ is the atom number density at a position $\vec{r}$ in the MOT. The integral is evaluated over the volume containing the trapped atoms. To measure the loss-rate coefficient $\beta$, the atom density distribution and number of atoms are measured at various times by absorption imaging, as described in [12]. There, we measured $\beta=1.75(35) \times 10^{-10} \mathrm{~cm}^{3} \mathrm{~s}^{-1}$ when $I_{t}=240 \mathrm{~mW} / \mathrm{cm}^{2}, \Delta_{11} /(2 \pi)=26 \mathrm{MHz}, \Delta_{22} /(2 \pi)=$ $12 \mathrm{MHz}$ and $B^{\prime}=48 \mathrm{G} / \mathrm{cm}$. Here, we present a more extensive study of how $\beta$ depends on intensity and detuning.

Figure 3(a) shows how $\beta$ depends on $I_{t}$. As $I_{t}$ is lowered from a high value, $\beta$ decreases in proportion, reaching a minimum of about $1.0 \times 10^{-10} \mathrm{~cm}^{3} \mathrm{~s}^{-1}$ when $I_{t}$ is between 50 and $100 \mathrm{~mW} / \mathrm{cm}^{2}$. At lower intensities, $\beta$ increases again, suggesting the onset of an extra loss mechanism. This dependence on $I_{t}$ is qualitatively similar to that measured for type-I MOTs $[16,17]$, but the variation we observe with $I_{t}$ is far smaller, and our values of $\beta$ are 10-100 times larger for all but the lowest of intensities used in the type-I MOTs. The loss rate coefficient for a type-I MOT operated at $\Delta \simeq \Gamma$ typically varies with intensity by two orders of magnitude [16], with the highest $\beta$ measured at the lowest intensities and attributed to hyperfine-state-changing collisions between ground-state atoms $[3,18,19]$ that release enough energy to eject the colliding atoms from the trap. A collision where only one atom changes hyperfine state imparts a kinetic energy of $\frac{1}{2} m v^{2}=$
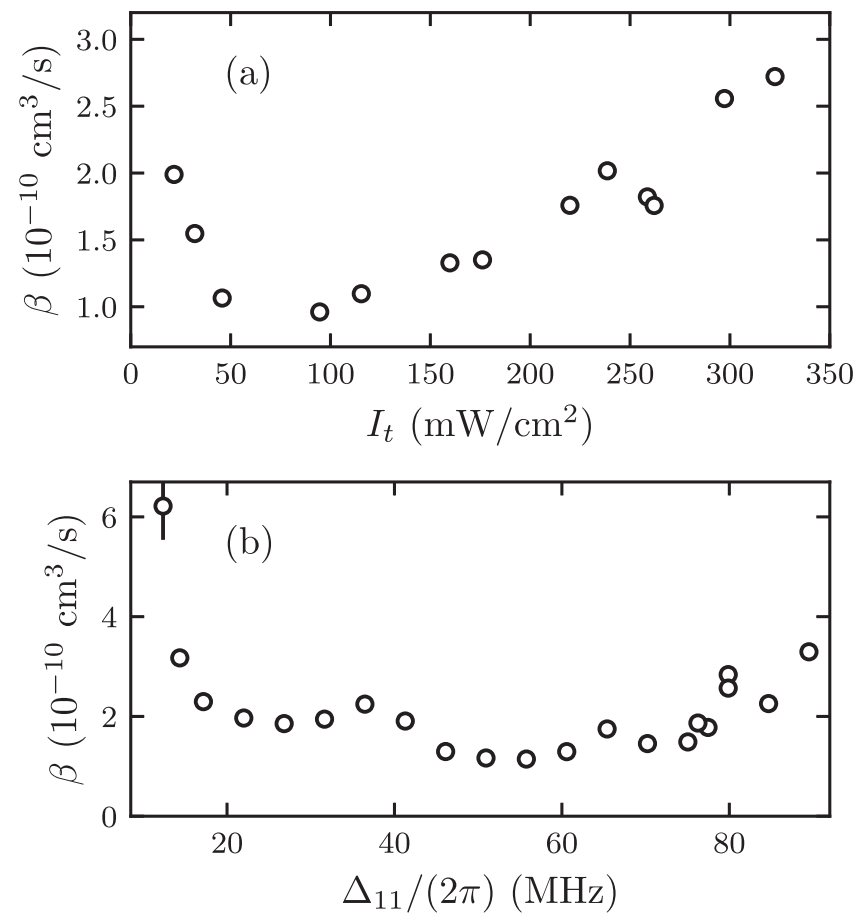

FIG. 3. Density-dependent loss rate coefficient, $\beta$, as a function of (a) trap laser intensity, $I_{t}$, and (b) detuning, $\Delta_{11}$. In (a) the other parameters are $\Delta_{22} /(2 \pi)=+12.5 \mathrm{MHz}, \Delta_{11} /(2 \pi)=+26 \mathrm{MHz}$, and $B^{\prime}=87 \mathrm{G} / \mathrm{cm}$. In (b) the other parameters are $I_{t}=215 \mathrm{~mW} / \mathrm{cm}^{2}$, $\Delta_{22} /(2 \pi)=+11.5 \mathrm{MHz}$, and $B^{\prime}=87 \mathrm{G} / \mathrm{cm}$.

$\Delta E_{\mathrm{hfs}} / 2$, where $\Delta E_{\mathrm{hfs}}=6.8 \mathrm{GHz}$ is the ground-state hyperfine splitting, giving $v=5.6 \mathrm{~m} / \mathrm{s}$. This is small compared to the capture velocity of a type-I MOT, except for low intensities, but exceeds the capture velocity we have measured in the blue-detuned MOT for all intensities (see Fig. 2). Therefore, the capture velocity of the blue-detuned MOT is insufficient to recapture the products of any inelastic collision between ground-state atoms, which probably explains the high value of $\beta$ we measure for all $I_{t}$, and the relatively small variation of $\beta$ with $I_{t}$. The additional loss mechanism we observe at low $I_{t}$ could be due to Zeeman-state-changing collisions in the wings of the distribution where the magnetic field is highest, or hyperfine-state-changing collisions involving excited-state atoms. The increasing value of $\beta$ with $I_{t}>50 \mathrm{~mW} / \mathrm{cm}^{2}$ may be due to the increased rate of collisions between atoms confined at a higher temperature, since $\beta=\langle\sigma(v) v\rangle$. We have previously measured a linear dependence of the temperature of the blue-detuned MOT on $I_{t}$ over this range [12].

Figure 3(b) shows how $\beta$ depends on the detuning $\Delta_{11}$. As this detuning is increased, $\beta$ is generally observed to fall, reaching its lowest value at $\Delta_{11} /(2 \pi) \approx 55 \mathrm{MHz}$. Interestingly, the capture velocity is also observed to decrease over a similar range of detuning, falling to approximately half its maximum value, as can be seen from Fig. 2(c). We might expect the loss rate to increase as the trap gets shallower, so the decreasing loss rate over this range of detuning cannot be a result of the changing trap depth. Instead, the decrease in $\beta$ may be due to a light-assisted process becoming less efficient or an optical shielding process becoming more efficient. Similar measurements have previously been made for 


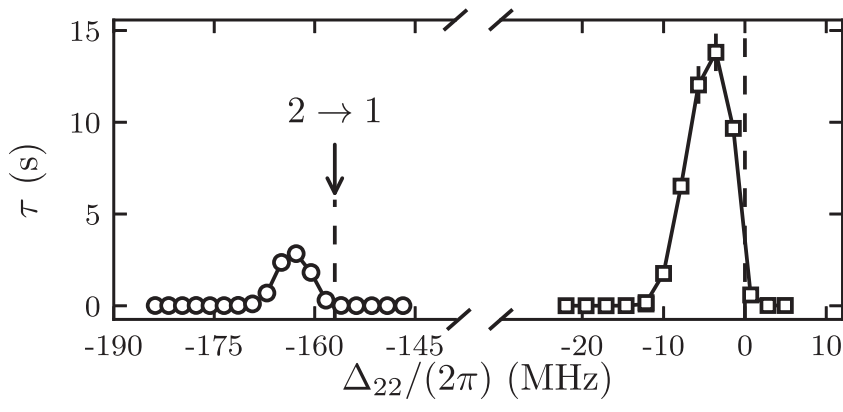

FIG. 4. The lifetime of atoms loaded into red MOT A as a function of $\Delta_{22}$. The frequency of the transition to $F^{\prime}=1$ is indicated. Two sets of data are shown. For each set $\mathcal{L}_{1}$ is tuned to maximise the lifetime of the MOT. For the circular markers this optimized detuning is $\Delta_{12} /(2 \pi)=-26 \mathrm{MHz}$ and for the square markers the detuning is $\Delta_{12} /(2 \pi)=-36 \mathrm{MHz}$. In both cases the total trap intensity is $I_{t}=260 \mathrm{~mW} / \mathrm{cm}^{2}$ and the field-gradient is $B^{\prime}=44 \mathrm{G} / \mathrm{cm}$.

type-II MOTs of $\mathrm{Na}$ and ${ }^{85} \mathrm{Rb}$. For a red-detuned type-II MOT of ${ }^{85} \mathrm{Rb}$, a value of $\beta=9.1(7) \times 10^{-9} \mathrm{~cm}^{3} \mathrm{~s}^{-1}$ has been reported [5], some 90 times larger than the minimum value we measure. The difference is partly attributable to the difference in temperatures between the two MOTs. Here, the temperature is about 30 times smaller than reported in [5], so atoms at a given density collide less frequently.

\section{RED-DETUNED MOTS}

Now we present observations of a number of trapping configurations that use red-detuned light. As shown in Fig. 1, these MOTs are grouped into pairs according to which excited-state hyperfine level $F^{\prime}$ the ground-state hyperfine level $F=1$ is coupled to.

\section{A. Red MOT A}

Red MOT A has a hybrid type-I/type-II nature. The restoring beams are polarised to drive $\Delta m_{F}=-1$ transitions, the same as for the type-I MOT. The $F=1$ ground state is coupled to $F^{\prime}=2$, so there are no dark ground states in this hyperfine level. Consequently, Doppler cooling can proceed more efficiently than in the pure type-II configurations. $\mathcal{L}_{2}$ can be red-detuned from $F^{\prime}=2$ or red-detuned from $F^{\prime}=1$, both of which give a trap lifetime of many seconds, as shown in Fig. 4. The lifetime is longest, up to $14 \mathrm{~s}$, when $\Delta_{22} /(2 \pi) \approx$ $-5 \mathrm{MHz}$. In this configuration, the MOT captures atoms from the background vapor, though the steady-state trapped atom number is much lower than for the type-I MOT. If a larger number of atoms is transferred from the type-I MOT into red MOT $\mathrm{A}$, the number of atoms decays towards the steady-state value. This initial decay is used to measure a trap lifetime. When $\mathcal{L}_{2}$ is tuned close to $F^{\prime}=2$, both ground states are coupled to the same excited state, which decays with equal probability to $F=1$ and $F=2$. The ground-state populations therefore tend to equalize. Because the detuning is negative, atoms in $F=2$ are heated by polarization-gradient forces. Figure 5 shows the temperature of red MOT A as a function of $I_{t}$. The temperature is measured from a sequence of absorption images recorded at fixed intervals during a

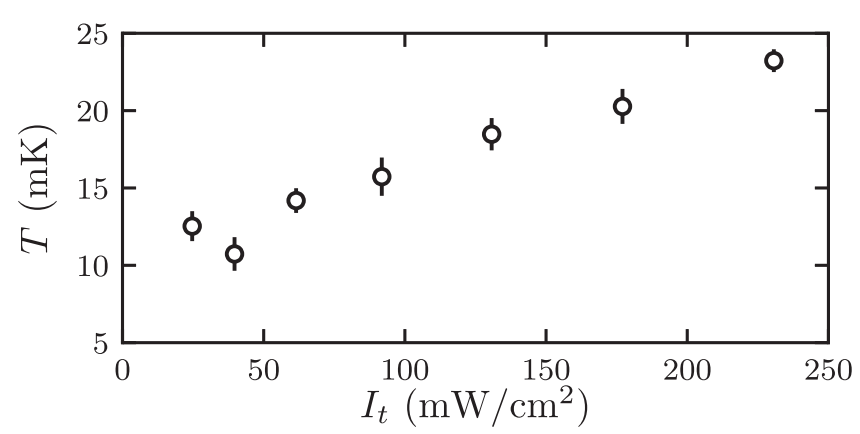

FIG. 5. The temperature of atoms confined in MOT A as a function of trap laser intensity. The other trap parameters are $\Delta_{12} /(2 \pi)=$ $-27 \mathrm{MHz}, \Delta_{22} /(2 \pi)=-4 \mathrm{MHz}$, and $B^{\prime}=44 \mathrm{G} / \mathrm{cm}$.

ballistic expansion, using Gaussian fits to the density distribution recorded in each image. The whole sequence is repeated five times. The temperatures measured here are orders of magnitude higher than those observed in the type-I MOT or blue-detuned type-II MOTs, but comparable to those observed before in red-detuned type-II atomic [5] and molecular MOTs [20].

\section{B. Red MOT B}

Now we discuss red MOT B, which is the red-detuned counterpart to the blue-detuned MOT. Here, the lasers are red-detuned from $F=1 \rightarrow F^{\prime}=1$ and $F=2 \rightarrow F^{\prime}=2$. The polarization of the MOT light is reversed relative to that used for a blue detuning in order to preserve a confining force. There is Doppler cooling, but atoms in both ground states are heated by polarization-gradient forces. We find that this MOT requires a large magnetic-field gradient and high laser intensity to be stable. Figure 6 shows a series of images showing atoms confined in the MOT as the intensity and field-gradient are varied. The image plane is parallel to the
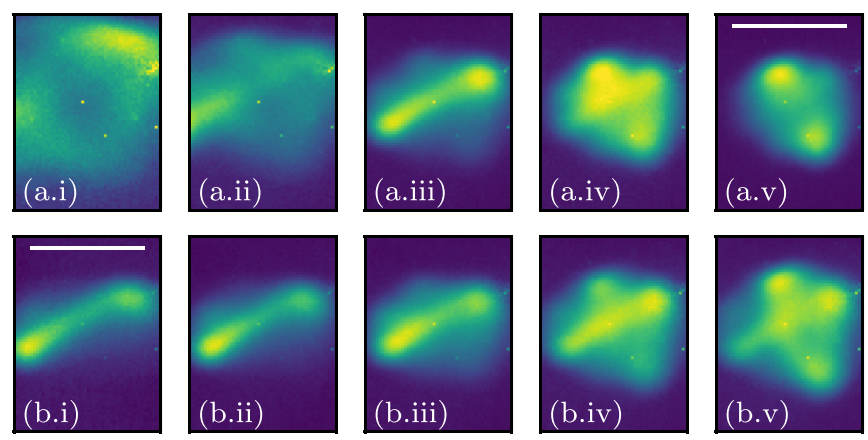

FIG. 6. Fluorescence images of atoms in MOT B. In the top row the magnetic-field gradient is increased from left to right. The field gradients used in (a.i)-(a.v) are $B^{\prime}=28,43,50,58$, and $65 \mathrm{G} / \mathrm{cm}$, respectively. The intensity is $I_{t}=103 \mathrm{~mW} / \mathrm{cm}^{2}$. In the bottom row the trap intensity is increased from left to right. The values in (b.i)(b.v) are $I_{t}=28,49,82,128$, and $165 \mathrm{~mW} / \mathrm{cm}^{2}$. The field gradient is $B^{\prime}=53 \mathrm{G} / \mathrm{cm}$. In all cases $\Delta_{11} /(2 \pi)=-25 \mathrm{MHz}$ and $\Delta_{22} /(2 \pi)=$ $-12 \mathrm{MHz}$. The solid white lines in (a.v) and (b.i) are scale bars of length $5 \mathrm{~mm}$. 
axis of the MOT and is aligned at $45^{\circ}$ to the radial beams. Fluorescence from the MOT is imaged onto a CCD camera during a $5 \mathrm{~ms}$ exposure. The cloud of atoms is very large in extent compared to the trapping configurations discussed so far, and striking ringlike structures are formed. Notably, atoms are always absent from the center of the MOT. The top row of images shows that increasing the field gradient causes the diameter of the ring structure to decrease and its orientation to change. The bottom row shows that increasing the intensity eventually causes a bifurcation into a double-ring structure. Similar behavior has been observed before in type-I MOTs, where a deliberate misalignment of the MOT beams can induce transitions between static and dynamic behavior. Both continuous ring structures and clumped orbital modes have been observed [21,22], both of which differ from the structures observed here by the presence of a central clump of atoms. This behavior in the type-I MOT was attributed to multiple photon scattering in the cloud. In the present investigation, the same MOT beams are used as in the other trapping configurations, where the density distributions are roughly Gaussian, so any misalignment must be small. However, since the temperature of the atoms in this MOT is so high, the trap volume explored by the atoms is much larger than typical. At the outermost regions of the trap a net torque is more readily imparted to the atoms by any small misalignment of the beams. We note that in red-detuned type-II MOTs, atoms are damped towards a special, nonzero velocity where the Doppler and polarization-gradient forces are in equilibrium [11]. One way to maintain this special speed is to move in circular trajectories, orbiting the trap center. We have performed trajectory simulations using the positiondependent and velocity-dependent force profiles calculated by solving the optical Bloch equations for this system, as described in [12]. These simulations show that an initially random distribution of atoms quickly forms itself into a ring with atoms circulating about the axis of strongest confinement, supporting our explanation for the structure we observe. The bifurcation to a double-ring structure could be due to the radiation pressure between atoms, which is not captured by our simple model.

We turn now to the lifetime of MOT B. For $B^{\prime}<50 \mathrm{G} / \mathrm{cm}$, trapped atoms form a diffuse cloud with a short lifetime. As $B^{\prime}$ is increased from this value, the confinement increases and the lifetime rises quickly, reaching a maximum of about $5 \mathrm{~s}$ at a field gradient of $65 \mathrm{G} / \mathrm{cm}$. At even higher $B^{\prime}$ the lifetime again begins to fall, possibly due to intratrap collisions. Figure 7 shows the MOT lifetime as the detuning of each of the lasers is scanned in turn. Figure 7(a) shows the lifetime as a function of $\Delta_{22}$, with $\Delta_{11} /(2 \pi)=-31 \mathrm{MHz}$. We find that $\mathcal{L}_{2}$ can be red-detuned from either $F^{\prime}=1$ or $F^{\prime}=2$, but the lifetime is many times higher in the latter case, acquiring a maximum value of $\tau \approx 6 \mathrm{~s}$. Figure 7(b) shows similar data as a function of $\Delta_{11}$, when $\Delta_{22} /(2 \pi)=-17 \mathrm{MHz}$. The lifetime of MOT B is largest when $-40 \leqslant \Delta_{11} /(2 \pi) \leqslant-30 \mathrm{MHz}$. We observe no MOT when $\Delta_{11} \approx-50 \mathrm{MHz}$, but the MOT reappears as $\mathcal{L}_{1}$ is tuned towards $F^{\prime}=0$. In this region of detuning, the maximum lifetime is about $1 \mathrm{~s}$, falling back to zero when the laser is resonant with $F^{\prime}=0$. When $\mathcal{L}_{1}$ is red-detuned from $F^{\prime}=0$, the lifetime quickly increases to high values and this MOT is referred to as red MOT C.
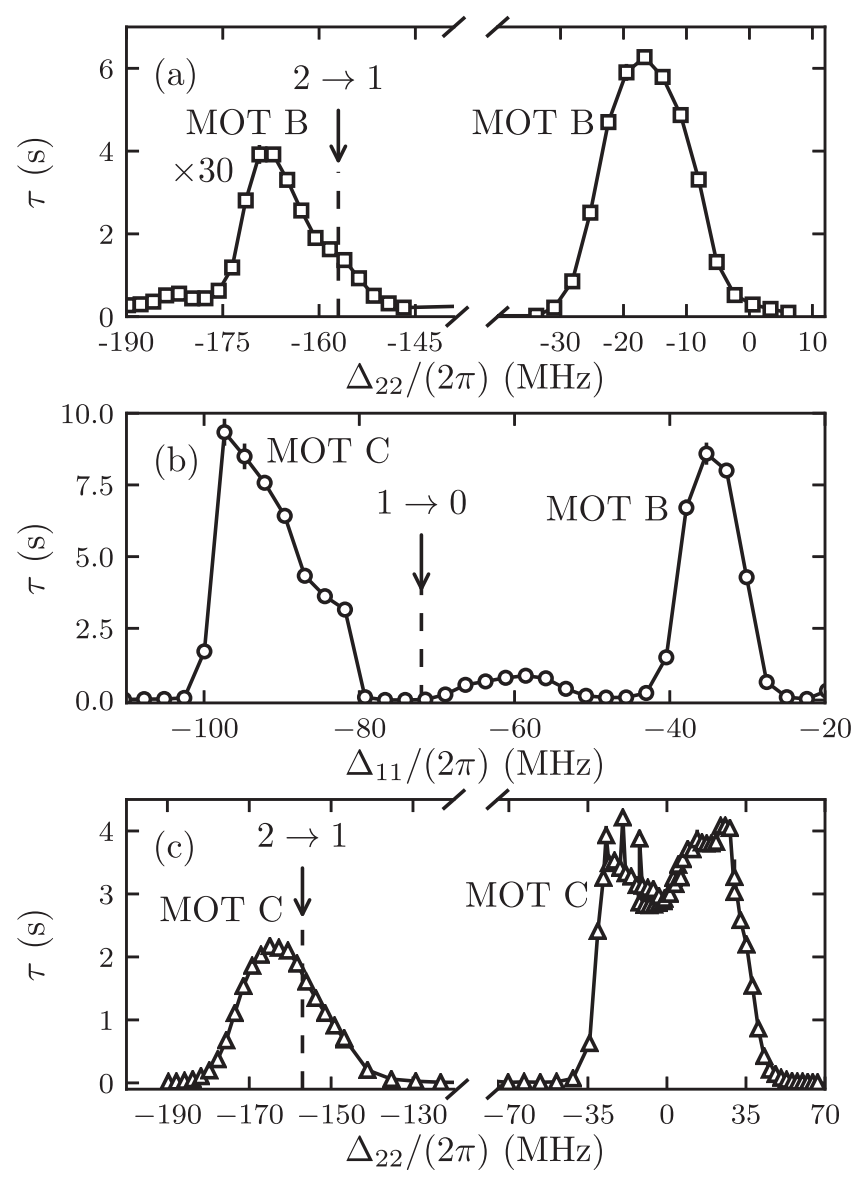

FIG. 7. The lifetimes of atoms loaded into MOTs B and C as a function of laser detunings. In (a) $\mathcal{L}_{2}$ is scanned as $\mathcal{L}_{1}$ is held fixed at $\Delta_{11} /(2 \pi)=-31 \mathrm{MHz}$. Then, in (b), $\mathcal{L}_{2}$ is fixed at $\Delta_{22} /(2 \pi)=-17 \mathrm{MHz}$ and $\mathcal{L}_{1}$ is scanned. MOT B appears when $-40 \leqslant \Delta_{11} /(2 \pi) \leqslant-30 \mathrm{MHz}$. As $\Delta_{11}$ is made more negative, two more regions of stable trapping appear that are red-detuned and bluedetuned relative to the $F=1 \rightarrow F^{\prime}=0$ transition. These are MOT C. A maximum lifetime for MOT C is obtained when $\Delta_{11} /(2 \pi)=$ $-97 \mathrm{MHz}$, which is equivalent to $\Delta_{10} /(2 \pi)=-25 \mathrm{MHz}$. Finally, with $\mathcal{L}_{1}$ fixed at $\Delta_{11} /(2 \pi)=-91 \mathrm{MHz}, \mathcal{L}_{2}$ is again scanned to obtain the data in (c).

\section{Red MOT C}

Red MOT C is the final trapping configuration discussed. This trap is obtained when $\mathcal{L}_{1}$ is tuned close to $F^{\prime}=0$ and $\mathcal{L}_{2}$ is tuned close to either $F^{\prime}=2$ or $F^{\prime}=1$, with the former giving longer lifetimes. There should not be any confining force when the excited state has no Zeeman splitting [10], so we would not expect $\mathcal{L}_{1}$ to contribute any confinement. Moreover, when $\mathcal{L}_{2}$ is tuned near $F^{\prime}=2$, we find that the lifetime is insensitive to the sign of $\Delta_{22}$, implying that $\mathcal{L}_{2}$ is not responsible for the confinement either. A similar observation has previously been reported for sodium [4], where the confinement was attributed to an alignment of the orientation of the ground-state atoms caused by a magnetic-field-induced asymmetry in the decay of atoms from $F^{\prime}=1$. The effect is the result of the mixing of excited-state hyperfine levels by the magnetic field. The force due to the magnetic-field-induced mixing is predicted to be proportional to the third power of 
the magnetic-field strength and inversely proportional to the hyperfine splitting. In ${ }^{87} \mathrm{Rb}$, the hyperfine spacing between $F^{\prime}=0$ and $F^{\prime}=1$ is $87 \mathrm{MHz}$, whereas in sodium the equivalent spacing is only $16 \mathrm{MHz}$. This could explain why MOT $\mathrm{C}$ is diffuse, being comparable in extent to the fluorescence images of MOT B shown in Fig. 6, despite the large field gradients used.

\section{SUMMARY, CONCLUSIONS, AND SUGGESTIONS}

For each of the alkali metal atoms there is just a single typeI MOT, and these have been studied extensively. There exist many more type-II trapping configurations, few of which have been reported previously. Here we have investigated a number of these configurations, and have presented their properties, which can vary enormously and sometimes be quite extreme. The configurations studied include both blue-detuned and reddetuned type-II MOTs. In these type-II MOTs, the Doppler and Sisyphus forces always have opposite signs, and the balance between these two forces dictate many of the properties of these MOTs. This work consolidates the recent progress made in understanding type-II MOTs, and verifies predictions about the conditions required for these MOTs [10,11].

In the blue-detuned MOT, Doppler heating dominates at high speeds but Sisyphus cooling dominates at low speed. Unlike in type-I systems, the Sisyphus cooling in type-II systems is robust to the magnetic field of the MOT, so strong cooling can occur in the blue-detuned MOT provided the atoms remain at low speeds. We have measured the capture velocity of the blue-detuned MOT as a function of laser detuning and intensity, finding values up to $3.8 \pm 0.1 \mathrm{~m} / \mathrm{s}$. Over the entire range of detunings and intensities explored, the capture velocity is at least ten times larger than the mean velocity of the atoms in the MOT. The atoms remain always at speeds where Sisyphus cooling dominates and so are cooled to low temperatures and are stable in the MOT despite the presence of Doppler heating at higher speeds.

We have presented a systematic study of the loss rate due to ultracold collisions in the blue-detuned MOT. We measure the loss rate coefficient, $\beta$, as a function of laser intensity and detuning. While the trend with intensity is similar to that observed in type-I MOTs, the variation is about 30 times smaller, and the value of $\beta$ is typically $10-100$ times larger. We suggest that the dominant mechanism is the release of energy in hyperfine-changing collisions between ground-state atoms. While this mechanism explains the high values of $\beta$ and the relatively small variation with intensity, it does not explain the increase in $\beta$ we observe at low intensities. This observation suggests that a new mechanism becomes active at low intensities, which may be Zeeman-state-changing collisions, or hyperfine-changing collisions between excited-state atoms. The dependence of $\beta$ on detuning shows a surprising structure which calls for further investigation, either experimental or theoretical. It may be that light-assisted collisional processes or optical shielding processes are playing a role.

We have also demonstrated the red-detuned counterpart of the blue-detuned MOT. This MOT is only stable at high intensity and high magnetic field gradient, and the temperature of the atoms is about 1000 times higher. In such red-detuned type-II MOTs, atoms are driven towards an equilibrium speed, typically a few $\mathrm{m} / \mathrm{s}$, where the Doppler and Sisyphus forces are balanced. One way to maintain this equilibrium speed, while remaining trapped, is to orbit around the center of the MOT. We indeed observe large ring structures, with no density at the center, consistent with this idea. The rings are stable, and we measure MOT lifetimes up to $6 \mathrm{~s}$, showing that the atoms can make thousands of orbits in a stable manner.

In the absence of Zeeman splitting in the excited state, there is no magneto-optical force [10]. Nevertheless, we observe a MOT which appears to involve the structureless $F^{\prime}=0$ excited state. We attribute this to mixing of the excited-state hyperfine levels by the magnetic field, as observed previously in sodium. The relevant excited-state hyperfine interval in ${ }^{87} \mathrm{Rb}$ is five times larger than in $\mathrm{Na}$, so we would expect the confining forces to be five times weaker for the same magnetic field gradient. While this MOT is indeed diffuse, it has a lifetime of up to $10 \mathrm{~s}$, suggesting that the mechanism is robust and would probably work for all the alkali metal atoms. It may also play a significant role in molecular MOTs of $\mathrm{SrF}$ and $\mathrm{CaF}$, where the relevant excited-state hyperfine interval is about 30 times smaller than here.

MOTs equivalent to those reported here should also be realizable for other laser-cooled alkali metal atoms, especially in cesium, where the hyperfine intervals are largest. For the lighter elements, where the hyperfine intervals are smaller, competing trapping and antitrapping MOT forces from neighboring excited-state levels may result in some of the configurations being unstable. More accurate predictions could be obtained from a rate-equation model of the full hyperfine structure of the atom, as in [10].

The various MOT configurations reported here have different distributions of populations among the ground-state sublevels and could be used to investigate collisions between trapped atoms in a variety of new settings. For example, it has previously been demonstrated that collisions between ground-state atoms can be optically shielded by blue-detuned light [23]. Conversely, red-detuned light can excite groundstate atoms to an attractive molecular potential, where they accelerate towards each other, approach at short distances, and may undergo an inelastic process leading to trap loss. In the $D_{2}$ line the excited state hyperfine intervals are small enough that colliding atoms in a blue-detuned MOT can still be excited to an attractive molecular potential of a higher-lying hyperfine state, and undergo inelastic collisions that eject them from the trap. However, a previous investigation has shown that trap loss due to light-assisted collisions is reduced by over an order of magnitude from its near-resonant value for a detuning $|\Delta|>800 \mathrm{MHz}$, which is about the size of the $D_{1}$-line hyperfine interval in ${ }^{87} \mathrm{Rb}$. Therefore, light-assisted collisions might be strongly suppressed in such a $D_{1}$ MOT, while the near-resonant blue-detuned light could optically shield inelastic collisions between ground-state atoms. Such a MOT could be formed using a similar procedure to that demonstrated here. The blue-detuned MOT could also be applied to molecules, where type-II transitions are always used. If that works, impressive increases in the phase-space density of molecular MOTs might be possible.

Underlying data may be accessed from the Zenodo repository [24] and used under the Creative Commons CCZero license. 


\section{ACKNOWLEDGMENT}

This research has received funding from EPSRC under Grants No. EP/M027716/1 and No. EP/ P01058X/1.

[1] E. L. Raab, M. Prentiss, A. Cable, S. Chu, and D. E. Pritchard, Phys. Rev. Lett. 59, 2631 (1987).

[2] L. G. Marcassa, K. Helmerson, A. M. Tuboy, D. M. Milori, S. R. Muniz, J. Flemming, S. C. Zílio, and V. S. Bagnato, J. Phys. B 29, 3051 (1996).

[3] S. Q. Shang, Z. T. Lu, and S. J. Freedman, Phys. Rev. A 50, R4449 (1994).

[4] K. Nasyrov, V. Biancalana, A. Burchianti, R. Calabrese, C. Marinelli, E. Mariotti, and L. Moi, Phys. Rev. A 64, 023412 (2001).

[5] V. B. Tiwari, S. Singh, H. S. Rawat, and S. C. Mehendale, Phys. Rev. A 78, 063421 (2008).

[6] J. F. Barry, D. J. McCarron, E. B. Norrgard, M. H. Steinecker, and D. DeMille, Nature (London) 512, 286 (2014).

[7] S. Truppe, H. J. Williams, M. Hambach, L. Caldwell, N. J. Fitch, E. A. Hinds, B. E. Sauer, and M. R. Tarbutt, Nat. Phys. 13, 1173 (2017).

[8] L. Anderegg, B. L. Augenbraun, E. Chae, B. Hemmerling, N. R. Hutzler, A. Ravi, A. Collopy, J. Ye, W. Ketterle, and J. M. Doyle, Phys. Rev. Lett. 119, 103201 (2017).

[9] E. S. Shuman, J. F. Barry, D. R. Glenn, and D. DeMille, Phys. Rev. Lett. 103, 223001 (2009).

[10] M. R. Tarbutt, New J. Phys. 17, 015007 (2015).

[11] J. A. Devlin and M. R. Tarbutt, New J. Phys. 18, 123017 (2016).

[12] K. N. Jarvis, J. A. Devlin, T. E. Wall, B. E. Sauer, and M. R. Tarbutt, Phys. Rev. Lett. 120, 083201 (2018).
[13] C. Monroe, W. Swann, H. Robinson, and C. Wieman, Phys. Rev. Lett. 65, 1571 (1990).

[14] K. E. Gibble, S. Kasapi, and S. Chu, Opt. Lett. 17, 526 (1992).

[15] D. Hoffmann, S. Bali, and T. Walker, Phys. Rev. A 54, R1030 (1996).

[16] C. D. Wallace, T. P. Dinneen, K.-Y. N. Tan, T. T. Grove, and P. L. Gould, Phys. Rev. Lett. 69, 897 (1992).

[17] S. D. Gensemer, V. Sanchez-Villicana, K. Y. N. Tan, T. T. Grove, and P. L. Gould, Phys. Rev. A 56, 4055 (1997).

[18] N. W. M. Ritchie, E. R. I. Abraham, Y. Y. Xiao, C. C. Bradley, R. G. Hulet, and P. S. Julienne, Phys. Rev. A 51, R890 (1995).

[19] R. S. Williamson and T. Walker, J. Opt. Soc. Am. B 12, 1393 (1995).

[20] H. J. Williams, S. Truppe, M. Hambach, L. Caldwell, N. J. Fitch, E. A. Hinds, B. E. Sauer, and M. R. Tarbutt, New J. Phys. 19, 113035 (2017).

[21] D. Felinto, L. G. Marcassa, V. S. Bagnato, and S. S. Vianna, Phys. Rev. A 60, 2591 (1999).

[22] T. Walker, D. Sesko, and C. Wieman, Phys. Rev. Lett. 64, 408 (1990).

[23] V. Sanchez-Villicana, S. D. Gensemer, K. Y. N. Tan, A. Kumarakrishnan, T. P. Dinneen, W. Süptitz, and P. L. Gould, Phys. Rev. Lett. 74, 4619 (1995).

[24] K. N. Jarvis, B. E. Sauer, and M. R. Tarbutt, Zenodo (2018), doi:10.5281/zenodo.1317582. 